\title{
El Enfoque Histórico-Cultural como estrategia en la formación de Pedagogos en una Universidad Privada de San Luis Potosí, México
}

\author{
The Historical-Cultural Approach as a strategy in the formation of \\ Pedagogues in a Private University of San Luis Potosí, Mexico
}

\section{Mónica Guadalupe Hernández Tovar ${ }^{1}$ Montserrat Cañedo Loredo}

\begin{abstract}
RESUMEN
En el siguiente artículo se presenta una pesquisa teórica que sustenta el aprendizaje social basado en el Enfoque HistóricoCultural, iniciado por Vygotsky, con la cual trabaja desde una Didáctica Desarrolladora con los estudiantes de la Licenciatura en Pedagogía en la Universidad Tangamanga, Campus Tequis en San Luis Potosí, México. Estas ideas brindan al docente la posibilidad de generar, desde el aula donde trabaja, las situaciones precisas que fomenten el aprendizaje colaborativo a través de una orientación activo-transformadora para la adquisición del conocimiento y los aprendizajes reales en los estudiantes.
\end{abstract}

Palabras clave: Enfoque Histórico-Cultural. Didáctica Desarrolladora. Aprendizaje colaborativo.

\begin{abstract}
The following article presents a theorical research that supports the social learning based on the Historical-Cultural Approach, initiated by Vygotsky, which he works from a Didactic Development with the students of Pedagogy at the Tangamanga University, Campus Tequis in San Luis Potosí, Mexico. These ideas give the teacher the possibility of generate, from the classroom where he works, the precise situations that enable collaborative learning through as active-transforming orientation for the acquisition of knowledge and real learning in students.
\end{abstract}

Keywords: Historic-Cultural Approach. Didactic Development. Collaborative learning.

\section{Introducción}

El objetivo de este artículo es la divulgación del impacto que tiene el enfoque Histórico-Cultural de Vygotsky en un contexto universitario.

Actualmente, la educación de jóvenes en las universidades presenta diversas problemáticas que se han ido trazando conforme la evolución de las

\footnotetext{
1 Maestra en Educación Basada en Competencias. Coordinadora de Pedagogía Escolar y Posgrados del Campus Tequis, de la Universidad Tangamanga. San Luis Potosí, México. Contacto: mhernandez@utan.edu.mx

2 Maestra en Educación con Especialidad en Docencia. Docente del Campus Tequis, de la Universidad Tangamanga. San Luis Potosí. México. Contacto: montse.canedo@hotmail.com
} 
Tecnologías de la Información y las Comunicaciones (TIC), ha incrementado y se ha ido integrando a la escuela. Esto ha propiciado que los alumnos se vuelvan cada vez más exigentes con los docentes y busquen siempre que el trabajo en el aula sea a través de las TIC.

Consideran que la educación es una mercancía para ser adquirida y consumida. Por eso esperan que su acercamiento al conocimiento sean lo más rápido, entretenido y sencillo posible al tiempo que buscan maximizar la relación tiempo de estudio/resultados obtenidos: aspiran a reducir lo más posible el primero al tiempo que incrementan los segundos. Son jóvenes que prefieren la práctica a la teoría, las tareas grupales a las individuales y la información en formato digital a los libros. (IBAÑEZ et al. 2008, p.3)

Lo anterior, ha propiciado que hoy en día se den una serie de transformaciones en las Universidades, como señalan TAGLIBAUE \& CUESTA, (2011) "Para adaptarse a la generación Millenial, las instituciones universitarias se han visto obligadas a modificar sus programas de estudio, sus servicios y asignaturas".

Se debe reconocer que entre los jóvenes de hoy en día, existe una inmensa capacidad multitareas, en la que, en el salón de clases pueden estar inmersos en el celular, a la vez que escuchan al docente impartir su cátedra y escuchan a su compañero de junto, además, la mayoría de sus actividades están enfocadas al logro de metas precisas (terminar la universidad, conseguir empleo o emprender un negocio). Aunado a lo antes mencionado, los alumnos actuales, muestran una gran disposición para trabajar en equipos colaborativos, que sean de preferencia en grupos de pares.

El trabajo en grupos de pares se ha señalado en el Enfoque HistóricoCultural, iniciado por Vygotsky (1930-1934), quien ha sido uno de los más grandes influenciadores que ha tenido el Constructivismo. Las principales investigaciones que Vygotsky realizó, se enfocaron en:

- Problemas teóricos y metodológicos de la Psicología

- Problemas de Psicología General

- Problemas del desarrollo de la psique 
- Psicología infantil

- Fundamentos de defectología

Estas investigaciones son importantes aun en la actualidad, ya que brindan a los profesionales de la educación un amplio panorama de estrategias para la mejor adquisición de conocimientos en los alumnos.

Para continuar, es preciso plantear la siguiente pregunta: ¿Cómo abordar el Enfoque Hístórico-Cultural en un contexto universitario preciso?

Los jóvenes de la Licenciatura en Pedagogía, de la Universidad Tangamanga, Campus Tequis, se encuentran en un contexto que promueve un aprendizaje significativo, ya que cuentan con aulas equipadas con todo lo que a tecnología se refiere, la tarea a trabajar por los docentes es el desarrollo de las clases con diversas estrategias innovadoras para la mejor adquisición de conocimientos y la producción de las habilidades y competencias necesarias en los alumnos, para llevar a cabo una práctica pedagógica entre grupos de pares.

\section{Enfoque Histórico Cultural}

Lev Vygotsky (1896-1934) fue un importante psicólogo ruso que, llevó a cabo, diversas investigaciones sobre el desarrollo cognitivo, las cuales han sido el parteaguas para desarrollar múltiples teorías acerca del conocimiento y el aprendizaje adquirido a través de la interacción social, lo cual recibe el nombre de: Teoría histórico-cultural del Desarrollo.

Este enfoque nace en Rusia a comienzos del Siglo XX, como una respuesta a la corriente Conductista (PAVLOV, I. Conditioned reflexes. 1926), Vygotsky (2009) plantea la idea de que:

Antes de llegar a dominar su propia conducta, el niño comienza a dominar su entorno con la ayuda del lenguaje. Ello posibilita nuevas relaciones con el entorno además de la nueva organización de la propia conducta. La creación de estas formas de conducta esencialmente humanas produce más adelante el intelecto, convirtiéndose, después, en la base del trabajo productivo: la forma específicamente humana de utilizar las herramientas. (VYGOTSKY, 2009, p. 48). 
La idea central del Enfoque Histórico-Cultural es resaltar la interacción existente de los factores sociales, históricos, culturales e individuales como pieza clave del desarrollo humano. (TUDGE, SCRIMSHER, 2003)

La forma en que el estudiante se relaciona con el mundo que le rodea (personas y objetos) transformará su visión y su pensamiento, pues el significado que le dé a cada proceso será significativo en medida a la vinculación que tenga con el. Existen además, algunos otros elementos que ayudarán al alumno a aprender, tales como: las ideas familiares arraigadas, la genética o herencia y las ideas particulares que el aprendiz irá desarrollando como individuo.

Respecto a lo anterior, Vygotsky (2000) comenta:

El desarrollo cultural del niño representa un tipo especial de desarrollo, es decir, el proceso de arraigo del niño en la cultura no puede identificarse con el proceso de madurez orgánica, por una parte, ni reducirse , por otra, a una simple asimilación mecánica de ciertos hábitos externos. (VYGOTSKY, 2000, p. 315).

\section{Enfoque Histórico-Cultural en la Universidad}

Las universidades requieren contribuir a la formación de profesionistas desde la realidad y necesidades del mundo actual, siendo importante tomar en cuenta el contexto de los alumnos para propiciar su desarrollo y potenciar sus capacidades, en este sentido se precisa en el enfoque histórico-cultural de Vygotsky.

El proceso de aprendizaje es una manera de apropiación de la herencia cultural disponible dada en las interacciones sociales del alumno en su entorno personal, social y académico que le permite adquirir los aprendizajes. (VYGOTSKY, 2009). Es por tanto prioritario que las universidades estudien el contexto cultural de los alumnos para lograr un aprendizaje que dé respuesta al ámbito profesional de los alumnos.

En este sentido el desarrollo del estudiante está ligado con su interacción en el contexto socio histórico cultural, para Vygotsky (1981)

El desarrollo ontogenético del ser humano tiene la siguiente estructura: actividad colectiva y comunicación-cultura (signos) 
apropiación de la cultura (enseñanza y educación)-actividad individual-desarrollo psíquico del individuo. (VYGOTSKY, 981, p. $63)$.

Partir de estos aspectos hace necesario realizar actividades de trabajo colaborativo entre los alumnos sobre proyectos relacionados con su profesión.

Las universidades tienen que desarrollar habilidades para que los estudiantes tengan la capacidad de aprender a lo largo de la vida, propiciando su autonomía; por lo que señala Zilberstein (2000):

La enseñanza debe considerarse inseparablemente vinculada a la educación y por consiguiente a la formación de una concepción determinada de mundo y de la vida, teniendo como objetivo el adquirir el dominio de los conocimientos acumulados en la experiencia cultural. (ZILBERSTEIN, 2000, p.29)

Por lo que, la enseñanza en las universidades debe estar vinculada a la actividad práctica del estudiante para la adquisición de conocimientos y su aplicación en el contexto personal y profesional que lo lleve a transformar e innovar positivamente su realidad. Vygotsky (1981), señala que:

El aprendizaje es una actividad social y no solo un proceso individual, que lleva a una actividad de producción y reproducción del conocimiento, mediante el cual el estudiante adquiere los modos sociales de acción e interacción. (VYGOTSKY, 1981, p.61)

Es por consiguiente que en el nivel universitario se vinculen los conocimientos teóricos con actividades relacionadas con su profesión, que le permitan apropiarse de los modos de actuación de su contexto profesional.

\subsection{Interacción docente-alumno}

En los procesos de enseñanza aprendizaje es trascendental que exista una buena comunicación maestro-alumno, que permita al docente dar la ayuda pedagógica que el alumno requiera, tal como como lo señala Vygotsky (2009):

El estudiante tiene una zona de desarrollo real, que es lo que sabe hacer por sí solo, mostrando sus capacidades y por otro lado si el docente u otro compañero más capaz, le da la ayuda para resolver un problema y lo soluciona, constituye la zona de desarrollo potencial, lo que el estudiante puede hacer con la ayuda de otros, esto se nombre zona de desarrollo potencial. (VYGOTSKY, 2009, p. 
133).

Es a partir de lo anterior que la interacción docente alumno propicia el aprendizaje y la adquisición de habilidades que le permitirán al alumno desempeñarse exitosamente tanto en el ámbito personal como en el profesional.

El nivel real de desarrollo evidencia la resolución independiente de un problema, define las funciones que ya han madurado, caracterizando el desarrollo mental que ya existe y la zona de desarrollo próximo define las funciones que aún no ha madurado, pero que se encuentran en proceso de maduración que puede lograr el alumno. En este sentido la relación de comunicación docente alumno permite el desarrollo y maduración de su desarrollo cognitivo dando la oportunidad un crecimiento personal y profesional.

Otro aspecto que establece Vygotsky (1981) es la interacción aprendizaje y desarrollo; precisando que el aprendizaje se da en dos planos, primero en el social y luego en el psicológico, dándose primero entre sus compañeros, maestros que es lo interpsicológico y luego en lo individual dentro del estudiante, que es lo intrapsicológico.

De esta manera nos muestra que el aprendizaje estimula y activa una variedad de procesos mentales que se dan en la relación de interacción con otras personas que ocurre en diferentes contextos y es mediada por el lenguaje. Esto hace importante el establecer formas de trabajo colaborativo entre los estudiantes, que los lleve a establecer esta ayuda pedagógica al elaborar sus proyectos o trabajos académicos, además de la guía, así como la interacción con el docente.

\subsection{Aprendizaje colaborativo mediante trabajo en grupos}

Retomando el núcleo del Enfoque Histórico-Cultural, es importante mencionar que el proceso de enseñanza aprendizaje fuera y dentro del aula es originado por la interacción entre un sujeto y un objeto de estudio, en donde la influencia del contexto y la cultura determinan el grado de aprendizaje. Es decir, el estudiante no es un individuo solitario, forma parte de una sociedad y está 
viviendo en un momento histórico preciso, por lo tanto, en el proceso de construcción del aprendizaje el docente debe entender, que el alumno no aprende solo y por su cuenta.

El ser humano es un ser social por naturaleza, por ende, el aprendizaje depende en gran parte de lo que observa, vive y comparte día tras día con las personas que le rodean. Se aprenden no sólo conceptos académicos, si no que, al vivir en sociedad, está determina los valores, costumbres y creencias con las que se debe desarrollar un sujeto desde que nace hasta que muere.

La Universidad debe promover el trabajo colaborativo en sus aulas, donde se logre una interacción entre los compañeros que fortalezca sus capacidades intelectuales, de acuerdo a J. Zilberstein. (2000). El aprendizaje se produce a través de una interacción social y, desde ese punto de vista, es un proceso social, tanto por sus contenidos como por las formas en que se genera. Un estudiante aprende de sus compañeros y con otros compañeros; en esa interacción desarrolla $\mathrm{su}$ inteligencia práctica y reflexiva, construyendo y asimilando nuevos conocimientos o representaciones mentales en su proceso de aprendizaje. De esta forma, los primeros favorecen la adquisición de otros y así sucesivamente.

De esta manera se puntualiza la relevancia y trascendencia que tiene el propiciar el trabajo colaborativo en las universidades, sustentado en la interacción social que se realiza en las aulas diariamente al trabajar en grupo o equipos, que les permitirá interiorizar los conocimientos y aplicarlos en situaciones reales.

\subsection{Tecnología en el aula}

En el ámbito de la educación superior el uso de las tecnologías se ha convertido en una herramienta básica para el desarrollo de los procesos de enseñanza aprendizaje, debido a las demandas del mundo globalizado y la evolución de la sociedad.

La utilización de las Tecnologías de la Información y Comunicación (TIC) con enfoque educativo ha permitido ampliar nuevas herramientas para el proceso de enseñanza aprendizaje, ya que permite al alumno acceder a una gran cantidad 
de información por medio de formas diversas como artículos de internet, videos, internet, bibliotecas virtuales, plataformas educativas virtuales, entre otros. El uso de TIC en el ámbito universitario permite que se establezca una interacción entre docentes y alumnos mediante estas tecnologías, que sirven como mediadores entre el profesor y sus estudiantes.

La actividad y comunicación del proceso educativo es optimizada y mediado por las TIC para su abordaje y asimilación, fundamentado en tareas que estimulen el aprendizaje, posibilitando trabajar la Zona de Desarrollo Próximo (ZDP) a partir de determinar las actividades a realizar, la organización, cómo las harán, con quienes, la regulación, propiciando trabajar la ZDP, para realizarlo se requiere de acciones que debe realizar el docente para el logro del objetivo de aprendizaje.

Los profesores deben orientar a los estudiantes a usar adecuadamente las tecnologías para su autoaprendizaje, ya que hoy en día los estudiantes para realizar una tarea consultan bases de datos especiales, bibliotecas virtuales, softwares educativos, etc. siendo medios que le permitirán elaborar trabajos de calidad. El aprendizaje desarrollador logra que el estudiante desarrolle su autonomía y autoperfeccionamiento en conexión con los procesos de socialización, compromiso y responsabilidad social. (SILVESTRE Y ZILBERSTEIN, 2002)

La implementación de las TIC requiere propiciar un aprendizaje activo y regulado, donde el estudiante construya sus conocimientos, por tanto se hace necesario planificar el uso de los medios tecnológicos de acuerdo al contexto de los alumnos.

\subsection{Estrategias aplicadas: Didáctica Desarrolladora}

La Didáctica Desarrolladora propone un Modelo de Enseñanza-Aprendizaje centrado en el alumno "es desarrolladora, en la misma medida que la enseñanza promueva el desarrollo integral de la personalidad del estudiante" (ZILBERSTEIN Y OLMEDO, 2015, p.64; SILVESTRE Y ZILBERSTEIN, 2002). Este desarrollo tendrá que ser desde la propia historia del educando, la cual está 
inserta en una cultura y con un lenguaje propio, es decir histórico cultural (VIGOTSKY, 1981).

En relación a las estrategias didácticas y su uso Zilberstein y Silvestre (2005, p.133) precisan que:

Implica acciones y operaciones que se emplean por cada sujeto, al realizar una determinada actividad o tarea, en este sentido se requiere que los docentes implementen en su práctica educativa estrategias que propicien el aprendizaje de los alumnos. (ZILBERSTEIN Y SILVESTRE, 2005, p. 133).

Por tanto, es importante que el maestro utilice una variedad de estrategias conociendo la función que tienen y cómo pueden implementarse. De acuerdo a Díaz y Hernández (2010, p. 119-121) se deben considerar los siguientes puntos:

- Insertar las actividades que realizan los alumnos, dentro de un contexto y objetivos más amplios donde están tengan sentido.

- Fomentar la participación e involucramiento de los alumnos en las diversas actividades y tareas.

- Realizar, siempre que sea posible, ajustes y modificaciones en la programación más amplia (de temas, unidades, etc.) y sobre la marcha, partiendo siempre de la observación del nivel de actuación que demuestren los alumnos en el manejo de las tareas y/o de los contenidos por aprender.

- Hacer uso explícito y claro del lenguaje, con integración de promover las situaciones necesarias de intersubjetividad (entre docente y alumnos), así como compartición y negociación de significados en el sentido esperado, procurando con ello evitar rupturas e incomprensiones en la enseñanza.

- Establecer constantemente relaciones explicitas y constante entre lo que los alumnos ya saben (sus conocimientos previos) y los nuevos contenidos de aprendizaje.

- Promover como fin último el uso autónomo y autorregulado de los contenidos por parte de los alumnos.

- Hacer uso del lenguaje para recontextualizar y reconceptualizar la experiencia pedagógica.

- Se considera fundamental la interacción entre alumnos, como otro recurso valioso para crear ZDP. (DÍAZ Y HERNÁNDEZ, 2010, p.119-121).

Por lo que el proceso de enseñanza aprendizaje implica y exige el uso de estrategias didácticas, como menciona Zilberstein y Silvestre (2005)

Las estrategias de aprendizaje son procedimientos para que los estudiantes se apropien en la actividad y comunicación de los conocimientos y desarrollo de habilidades ya que son recursos de autorregulación que le permiten lograr su aprendizaje. 
(ZILBERSTEIN Y SILVESTRE, 2005, p.68)

En las aulas se debe implementar y estimular el desarrollo de estrategias que le permitan al estudiante el lograr de su aprendizaje, para tener la solidez para la asimilación de conocimientos, habilidades y formación de valores.

\subsection{Zona de Desarrollo Próximo}

VYGOTSKI (2009), definió a la Zona de Desarrollo Próximo de la siguiente manera:

No es otra cosa que la distancia entre el nivel real de desarrollo, determinado por la capacidad de resolver independientemente un problema, y el nivel de desarrollo potencial, determinado a través de la resolución de un problema bajo la guía de un adulto o en colaboración con otro compañero más capaz. (VYGOTSKI, 2009,133)

Este término se puntualiza de dos formas:

- Es la distancia en el nivel real de desarrollo, determinado por la capacidad de resolver independientemente un problema, y el nivel de desarrollo potencial, determinado a través de la resolución de un problema bajo la guía de un adulto o en colaboración con otro compañero más capaz.

- Define aquellas funciones que no han madurado, pero que están en proceso de maduración.

La Zona de Desarrollo Próximo es de suma importancia en el proceso de enseñanza-aprendizaje, debido a que involucra el nivel de desarrollo que no está fijo. Se establece una gran diferencia entre lo que una persona puede realizar sola y lo que puede llegar a lograr con la ayuda de un tutor o compañero que va más avanzado.

Es trascendental, que el docente tenga claro que es él, el principal responsable del aprendizaje de los alumnos y que comprenda que este está relacionado con el desarrollo y la interacción social. Para esto, ONRUBIA (1999, P. 109-114) propone ocho puntos, de los cuales se toman los siguientes cuatro 
para que una Zona de Desarrollo Próximo sea más eficaz y exitosa en la Universidad:

1. Insertar en el máximo grado posible, la actividad puntual que el alumno realiza en cada momento en el ámbito de marcos u objetivos más amplios en los cuales esa actividad pueda tomar significado de manera más adecuada. (ONRUBIA, 1999, p. 109114)

Es decir que los alumnos más avanzados y mejor preparados definirán un marco global en el que las actuaciones del participante menos competente se inserten y tomen significado.

2. Posibilitar, en el máximo grado posible, la participación de todos los alumnos en las distintas actividades y tareas, incluso si su nivel de competencia, su interés o sus conocimientos resultan en un primer momento muy escasos y poco adecuados. (ONRUBIA, 1999, p. 109-114)

Habrá que sensibilizar y motivar a todo el grupo acerca de la importancia que tiene la contribución de ideas y conceptos de todos los involucrados en la clase y el cumplimiento de las tareas, aún si sus respuestas no son del todo atinadas. Para que así exista una actividad conjunta de todo el grupo.

3. Establecer un clima relacional, afectivo y emocional basado en la confianza, la seguridad y la adaptación mutuas, y en el que tengan cabida la curiosidad, la capacidad de sorpresa y el interés por el conocimiento por sí mismo. (ONRUBIA, 1999, p. 109-114).

Se debe recordar que, para que la Zona de Desarrollo Próximo pueda avanzar, no sólo importan los aspectos cognoscitivos e intelectuales, sino también hay que tomar en cuenta los de carácter relacional, afectivo y emocional. Hay que buscar esa conexión afectiva y el aprendizaje será más significativo. " 4 . Promover la utilización y profundización autónoma de los conocimientos que se están aprendiendo por parte de los alumnos.” (ONRUBIA, 1999, P. 109-114)

El último paso del proceso sería este, es decir, llegar a la interiorización: que el alumno haga propio el conocimiento adquirido de sus tutores. COLL (1999) menciona que existe una condición básica e irremplazable para que la enseñanza sea una verdadera ayuda en el proceso "Que se ajuste a la situación y 
características propias que en todo momento presenta la actividad mental de cada alumno".

La enseñanza, es decir la ayuda que brinde el docente, debe ir encaminada no a lo que el alumno ya conoce, sino hacia lo que el alumno no conoce y no domina, poniendo el docente un grado máximo de exigencia para que el alumno aprenda de una manera significativa.

\section{Conclusiones}

En este documento se presentaron diversas referencias de carácter teórico conceptual, que permitieron sustentar el desarrollo y la importancia del Enfoque Histórico-Cultural en el ámbito universitario, específicamente, en la formación de Pedagogos. En la Universidad Tangamanga, Campus Tequis, se brindan las facilidades necesarias para que los docentes lleven a cabo un trabajo constructivista en el aula, tales como:

- La utilización de TIC en el aula: computadora, pantalla, aplicaciones de internet que permiten un trabajo lúdico, por tanto resulta un aprendizaje significativo.

- Desarrollo de proyectos basados en el contexto actual al que se enfrentarán los alumnos.

- Comunicación cercana entre alumno-docente-coordinador.

- Elaboración de materiales didácticos de utilidad tanto para el docente como el alumno.

- Realización de actividades culturales enfocadas a la formación pedagógica.

- Participación en eventos de divulgación científica.

Las TIC permiten a los jóvenes universitarios desenvolverse en diversos escenarios que presentan el trabajo colaborativo entre pares como eje.

Retomando el Enfoque Histórico Cultural y la Zona de Desarrollo Próximo es importante recalcar que el docente, es el principal guía del proceso de enseñanza aprendizaje y que, de acuerdo con GARCÍA et al (2014):

Se deben tener presente dos conceptos claves del enfoque históricocultural que son la situación social del desarrollo, como punto de 
partida de todos los cambios dinámicos que se producen entre el joven y el entorno y el de zona de desarrollo próximo, concepto de suma importancia, pues permite determinar científicamente el desarrollo alcanzado por los estudiantes y cómo potenciarlo. (GARCÍA et al 2014, p.469)

Con lo que aquí se presentó, se trata de dar al Enfoque Histórico-Cultural una apertura a nuevos espacios, para continuar promoviendo la importancia de desarrollar en los alumnos el trabajo entre pares en los distintos escenarios en los que se desenvolverá de acuerdo al Perfil de Egreso que la Licenciatura en Pedagogía precisa.

\section{Referencias}

COLL, C., MARTÍN. E., MAURI, T., MIRAS, M., ONRUBIA, J., SOLÉ, I. \& ZABALA, A. El constructivismo en el aula. Barcelona: Graó, 1999.

DÍAZ, F., HERNÁNDEZ, G. Estrategias docentes para un aprendizaje significativo: una interpretación constructivista. Mc Graw Hill. 2010.

GARCÍA, M., VARELA DE MOYA, H., SIFONTES, B. \& PEÑA, M. Significación del enfoque histórico-cultural de Vygotsky para el tratamiento de las relaciones interdisciplinarias. Humanidades Médicas, 14 (2), 458-471. 2014. Recuperado el 16 de julio de 2018 , de http://scielo.sld.cu/scielo.php?script $=$ sci arttext\&pid $=\mathrm{S} 1727$ $\underline{81202014000200014 \& \operatorname{lng}=\text { es \&tlng=es. }}$

IBAÑEZ, E., CUESTA, M., TAGLIBAUE, R \& ZANGARO, M. La generación actual en la universidad: el impacto de los millennials. V Jornadas de Sociología de la UNLP. Universidad Nacional de La Plata. Facultad de Humanidades y Ciencias de la Educación. Departamento de Sociología. La Plata. 2008. Disponible en: https://www.aacademica.org/000-096/261.pdf , p.3

KOZULIN, A. The concept of activity in Soviet psychology: Vygotsky, his disciples and critics. American Psychologyst, 41. P. 264-274. 1986.

ONRUBIA, J. V. Enseñar: crear zonas de Desarrollo Próximo e intervenir en ellas. En: Coll, C. (Ed.), El constructivismo en el aula. Barcelona: Graó,1999.

SCHUNK, D. Teorías del aprendizaje. Una perspectiva educativa. México: Pearson educación, 2012. 
SILVESTRE, M., ZILBERSTEIN, J. Hacia una didáctica desarrolladora. Ciudad Habana: Pueblo y Educación. 2002.

TAGLIBAUE, R. \& CUESTA, M. Una Nueva Generación en la Universidad y el Trabajo: desafíos y oportunidades. Revista Gestión de las Personas y Tecnología, 4(12), p. 50-58. Chile. 2011.

TUDGE, J., SCRIMSHER, S. Lev s. Vygotsky on education: a cultural-historical, interpersonal, and individual approach to development. En: Zimmerman, B. \& SCHUNK, D. (Ed). Educational psychology: a century of contributions. P. 207228. Mahwah: Erlbaum. 2003.

VYGOTSKY, L. S. El desarrollo de los procesos psicológicos superiores. Barcelona: Crítica. 2009.

VYGOTSKY, L. S. Pensamiento y Lenguaje. Buenos Aires: La Pléyade. 1981.

WERTSCH, J. Culture, communication, and cognition: Vygotskian perspectives. Nueva York: Cambridge University Press. 1985.

J. ZILBERSTEIN. ¿Cómo hacer más eficiente el aprendizaje? México: Ediciones CEIDE. 2000.

ZILBERSTEIN, J.; OLMEDO, S. Didáctica Desarrolladora: posición desde el enfoque Histórico Cultural. Educação e Filosofia, Uberlândia, V. 29, n. 57, p. 61 93, jan./jun. 2015. ISSN 0102-6801. 2015.

\section{Monica Guadalupe Hernández Tovar}

Maestría en Educación Basada en Competencias

Licenciatura en Pedagogía

Coordinadora de la Licenciatura en Pedagogía Escolar y Posgrados en Psicología,

Docente de Licenciatura, Bachillerato, Secundaria y Primaria

\section{Montserrat Cañedo Loredo}

Doctorado en Ciencias de la Educación (en proceso de Titulación)

Maestría en Educación con Especialidad en Docencia

Licenciatura en Psicología

Docente de Licenciatura, Bachillerato, Media Terminal, Secundaria y Primaria 